\title{
Old and new therapeutic strategies in systemic sclerosis (Review)
}

\author{
CARMEN BOBEICA $^{1 *}$, ELENA NICULET $^{1}$, ALIN LAURENTIU TATU $^{2-4}$, MIHAELA CRAESCU $^{1 *}$, \\ DAN VATA $^{5}$, LAURA STATESCU ${ }^{5}$, ALINA VIORICA IANCU ${ }^{1,2}$, CARMINA LIANA MUSAT $^{1}$, \\ MIRUNA LUMINITA DRAGANESCU ${ }^{3}$, CRISTIAN ONISOR $^{1}$, MIHAELA LUNGU $^{3}$, SILVIA FOTEA $^{3 *}$, \\ AUREL NECHITA $^{3}$, BOGDAN IOAN STEFANESCU ${ }^{6}$ and LAURA GHEUCA-SOLOVASTRU ${ }^{5}$ \\ ${ }^{1}$ Department of Morphological and Functional Sciences, Faculty of Medicine and Pharmacy, 'Dunărea de Jos' University, \\ 800216 Galati; ${ }^{2}$ Multidisciplinary Integrated Center of Dermatological Interface Research MIC-DIR (Centrul Integrat \\ Multidisciplinar de Cercetare de Interfata Dermatologica - CIM-CID), 'Dunarea de Jos' University, 800010 Galati; \\ ${ }^{3}$ Clinical Medicine, Faculty of Medicine and Pharmacy, 'Dunărea de Jos' University, 800216 Galati; ${ }^{4}$ Research Center \\ in The Field of Medical and Pharmaceutical Sciences, ReFORM-UDJ, 800010 Galati; ${ }^{5}$ Clinical Department \\ and ${ }^{6}$ Department of Clinical Surgery, Faculty of Medicine and Pharmacy, 'Gr. T. Popa' University \\ of Medicine and Pharmacy, 700115 Iași, Romania
}

Received September 29, 2021; Accepted October 29, 2021

DOI: $10.3892 / \mathrm{etm} .2021 .11057$

\begin{abstract}
Systemic sclerosis (SSc) is a chronic inflammatory disease with autoimmune determinism having an incompletely known pathogenesis. Although not all links in the pathogenic chain are known, studies have shown that vasculopathy is the initial event and is followed by extensive fibrosis of the skin and internal organs. New therapeutic strategies have been developed in recent years, thanks to innovative research which has increased understanding of the disease mechanisms. No curative treatment for SSc is currently known. Therefore, the therapeutic target in SSc is its symptomatology. Peripheral
\end{abstract}

Correspondence to: Dr Elena Niculet or Dr Cristian Onisor, Department of Morphological and Functional Sciences, Faculty of Medicine and Pharmacy, 'Dunărea de Jos' University, 35 Alexandru Ioan Cuza Str., 800216 Galati, Romania

E-mail: helena_badiu@yahoo.com

E-mail: cristi.onisor@gmail.com

*Contributed equally

Abbreviations: SSc, systemic sclerosis; ACE, angiotensin converting enzyme; SRC, sclerodermal renal crisis; EULAR, European League against Rheumatism; MMF, mycophenolate mofetil; DLCO, diffusing capacity of the lungs for carbon monoxide; NT-proBNP: pro-B N-terminal natriuretic peptide; TGF- $\beta 1$, transforming growth factor- $\beta 1$; IL- 6 , interleukin-6; NO, nitric oxide; eNOS, endothelial nitric oxide synthetase; FGFR3, fibroblast growth factor receptor 3; PDGF, platelet-derived growth factor; VEGF, vascular endothelial growth factor; FVC, forced vital capacity; CB2, cannabinoid 2; EPCs, endothelial progenitor cells

Key words: systemic sclerosis, treatment, vasodilators, antifibrotics, immunosuppressants vasculopathy can be improved by administering vasodilators. Endothelin receptor antagonists and 5-phosphodiesterase inhibitors have a double benefit, both on peripheral and on pulmonary vasculopathy. Several molecules with antifibrotic effects are currently available; however, further studies are needed to confirm their beneficial effects. Immunosuppressants manage to control the cutaneous and visceral fibrotic process, thereby remaining as first-line drugs in the treatment of SSc. Although biological therapy using rituximab and tocilizumab has shown promising results in pulmonary fibrosis, ongoing studies are needed to determine their exact impact. The authors have differing views on the triggering role of glucocorticoids and the benefits of angiotensin-converting enzyme inhibitors in renal scleroderma. Some aspects of this disease such as calcinosis and pruritus, asthenia, or joint and muscle damage, remain difficult to manage.

\section{Contents}

1. Introduction

2. Immunosuppressive therapy

3. Treatment of digital microvasculopathy

4. Treatment of pulmonary hypertension

5. Treatment of skin fibrosis

6. Treatment of pulmonary fibrosis

7. Treatment of sclerodermal renal crisis

8. Treatment of esogastrointestinal damage

9. Conclusions

\section{Introduction}

Systemic sclerosis (SSc), also known as systemic scleroderma, is a collagenosis encumbered by a chronic inflammatory process with autoimmune determinism. The pathogenesis of 
this disease remains to be elucidated. Vasculopathy is the first step in its pathogenic chain, followed by an extensive skin and internal organ fibrosis. Research over the past two decades has increased understanding of this pathogenic process and new therapeutic strategies have been developed as a result (1). The premise for a favorable prognosis is a positive diagnosis and initiation of aggressive treatment as early as possible in disease evolution. Recent research discusses individualized, adapted treatment for each patient. Furthermore, deep insights into the pathogenic mechanisms underlying SSc have validated new treatments and have improved SSc management in general. Imunosuppressants and antifibrotic drugs have improved the course of the disease (2).

SSc treatment targets the symptoms of the disease rather than its pathogenesis, the last of which remains to be elucidated; thus, a curative treatment remains to be found. The 2017 updated SSc European League Against Rheumatism (EULAR) treatment guide sets clear treatment strategies according to the affected organ (3). SSc treatment is oriented towards digital peripheral microvasculopathy (represented by digital pulp ulcerations and Raynaud phenomena), as well as towards pulmonary hypertension, cutaneous fibrosis, pulmonary fibrosis, gastrointestinal affliction and sclerodermal renal crisis (SRC) (4).

Recent research has discussed individualized patient treatment, management personalization being required by the heterogeneous aspect of this disease and by the various patterns of multiorgan damage, guided by the predictable role of immunological markers (5). Progress made in recent years has resulted in the identification of certain links in the pathogenic chain and have led to increased survival rates through improved management of disease complications. However, some aspects of this disease, including musculoskeletal damage, asthenia, pruritus and calcinosis, are difficult to control. Although no curative treatment is known, improvement of vasculopathy and pulmonary fibrosis yields promising results (6). As low patient adherence has limiting effects on patient treatment, a new therapeutic approach aiming at epigenetic interaction, is required $(2,7)$.

\section{Immunosuppressive therapy}

Immunosuppressants are drugs used as a first-line treatment in SSc. Their beneficial effects are undeniable in improving skin hardening and lung damage. Of these, cyclophosphamide is indicated in repeated administrations, used predominantly in diffuse SSc. Despite its side effects, the beneficial effects of cyclophosphamide are maintained with the consecutive introduction of other immunosuppressant agents including methotrexate, azathioprine, or mycophenolate mofetil (MMF) (7-9). Cyclophosphamide is also effective in antral hemorrhage in the background of gastric vascular ectasia $(2,7)$.

The results on the efficacy of cyclophosphamide in diffusing capacity of the lungs for carbon monoxide (DLCO) recovery are divergent (2). Moreover, the risk of hemorrhagic cystitis, its teratogenic risk and the medullary suppressive effects, require caution and careful monitoring when used (10). The association between intravenous cyclophosphamide and oral azathioprine showed patient improvement in a 6-month British study (6).
A study conducted in 2018 did not have convincing results, being rendered inefficient, after the use of another immunosuppressant, tacrolimus, administered in combination with prednisolone $(2,11)$. With cyclophosphamide-like efficacy, an anti-CD20 monoclonal antibody, rituximab, was found to improve skin fibrosis and is thus used as an alternative to cyclophosphamide to avoid its side effects. However, for adequate pulmonary fibrosis control, the need to associate MMF as an immunosuppressant has been highlighted. Moreover, rituximab has net benefit in subcutaneous calcifications and vasculopathy (2). Tocilizumab, an anti-IL6 monoclonal antibody, appears to be more effective in the early stages of this disease, in lung dysfunction and in overlap syndrome; its effects have been found to be limited on cutaneous fibrosis $(2,6)$. Although tocilizumab and rituximab appear to be effective, more studies are required to verify their positive effects. The administration of these immunosuppressants is contraindicated when ulcers are infected. In their study, Hughes and Herrick (10) presented a clinical case study by Khor et al who revealed that, recurrent ulcers could heal after treatment with rituximab (11).

\section{Treatment of digital microvasculopathy}

The use of vasoactive agents is essential for the improvement of vasculopathy and implicitly for Raynaud's phenomenon, in preventing digital ulcerations and multiorgan damage $(2,12)$. The effectiveness of vasodilators cannot be disputed because it solves critical ischemia and prevents gangrene. Therefore, vasodilators can reduce the rate of amputations (3). The antiplatelet effect induced by the drug Aspenter did not prove a major benefit in resolving digital ulcers, nor for ameliorating the Raynaud phenomenon. However, antiplatelet agents are recommended in SSc. Moreover, anticoagulation with heparin does not appear to be useful and has a risk of gastrointestinal bleeding (13-16). Although pentoxifylline is an effective vasodilator that can relieve acral lesions, it cannot control the Raynaud's phenomenon. However, pentoxifylline functions as an immunomodulator by blocking TNF production and limits collagen synthesis by stimulating collagenase (17-19).

Endothelin receptor antagonists and 5-phosphodiesterase inhibitors are available for use in arterial pulmonary hypertension. Prostanoids such as iloprost, are indicated in Raynaud's phenomenon which is refractory to oral vasodilators (2).

Some authors emphasize the importance of physical and occupational therapy aimed at improving grasping and other hand functions (2). Of high interest is the approach of Fiori and his study team, who highlighted the favorable influence of pets and dogs on the perception of pain. The presence of the dog during iloprost infusion treatment aims at increasing treatment adherence and at reducing anxiety (20).

Calcitonin reduces Raynaud's phenomenon by blocking prostacyclin in the peripheral circulation. Infused dextran provides benefits in terms of antiplatelet aggregation and fibrinolysis but has the disadvantage of providing IgG and implicitly may pose the risk of triggering anaphylactic shock (21). N-acetylcysteine, an antioxidant administered long term for almost 3 years, has improved the healing rate of digital ulcers by reducing oxidative stress as well as the free radical levels. Digital ulcerations appear to evolve favorably under statin administration. Specifically, $40 \mathrm{mg}$ of atorvastatin 
administered over a 4-month period prevents new ulcer development $(12,13)$. When digital pulp ulcers progress unfavorably, surgical treatment may be a treatment option. Debridement of necrotic tissue and removal of pus relieves pain. The risk of complications to gangrene is $22.6 \%$ and occasionally amputation of the necrotic phalanx is inevitable $(12,13)$. Osteomyelitis requires magnetic resonance imaging for diagnosis and surgical treatment may be required. Pain can be controlled with opioids when it is resistant to analgesics (12).

\section{Treatment of pulmonary hypertension}

The PATENT-1 study, which enrolled 443 patients, evaluated the benefit of guanylate cyclase stimulator on pulmonary hypertension (22). Riociguat, administered for a period of 12 weeks as 3 daily doses of $2.5 \mathrm{mg}$ each, improved the walking test by $6 \mathrm{~min}$. Compared to the placebo group, the walking distance increased by 36 meters in the investigated group of patients. It is estimated that rociguat reduces dyspnea, improves exercise capacity and increases receptivity to endogenous nitric oxide (NO). As a result, rociguat has a decreasing effect on the vascular resistance from the pulmonary circulation and enhances the pro-B N-terminal natriuretic peptide (NT-proBNP) (22). Selexipag, an orally administered selective prostacyclin receptor agonist, was shown to be effective in the GRIPHON study. Selexipag reduced the complication rate by $39 \%$ compared to the placebo. The use of prostanoids is effective, but the side effects require taking numerous precautions (21).

Bosentan and sitaxentan treatment results are similar in SSc secondary arterial pulmonary hypertension, with a severity that is higher than in idiopathic pulmonary hypertension $(4,22,23)$. When bosentan is inefficient, sitaxentan may be another treatment option. Sitaxentan is another selective endothelin receptor antagonist with lower hepatotoxicity than bosentan (4).

The AMBITION study results revealed that the use of ambrisentan and tadalafil as a combination therapy was more effective than their individual use. This result is reflected by a lower hospitalization rate (of only 4\%) for the combination therapy, as compared to $12 \%$ for monotherapy. Nevertheless, further studies are required to identify the optimal drug combinations and their context of administration (21).

Remodeling of pulmonary capillaries in SSc imposes restrictions for the use of vasodilators. In SSc, vasodilator treatment may precipitate pulmonary edema associated with veno-occlusive disease (17). Occasionally, SSc can be associated with pulmonary thromboembolism and can determine an increase in the pulmonary artery pressure, a situation in which surgical treatment is appropriate and, in case of contraindication, a stimulator of guanylate cyclase, such as riociguat, can be administered $(21,24,25)$.

Nifedipine does not appear to be as effective as losartan in ameliorating Raynaud's vasculospastic seizures. In addition, losartan decreases transforming growth factor- $\beta 1$ (TGF- $\beta 1$ ) and has a favorable effect on cardiac and pulmonary fibrosis and improves the prognosis of SRC (17). Previous findings revealed that damaged endothelial cells produce excess NO and can reduce the level of endothelial nitric oxide synthetase (eNOS). As a result, a NO precursor, L-arginine, improves
Raynaud's phenomenon and has a favorable effect on blood circulation. This observation supports the usefulness of a NO-based gel with topical administration for improving cutaneous vascularization, or the use of inhaled administration of NO and prostacyclines having pulmonary vasodilating effects. The vasopressor effect in the pulmonary artery improves the prognosis of pulmonary hypertension (17).

Local treatment of ulcers can be complemented by adjuvant therapies, such as the use of negative subatmospheric pressure and hyperbaric oxygen therapy, which shrinks the skin lesion and brings the edges of the ulcerative lesion closer $(12,24,25)$. The use of acoustic shock waves activates cellular metabolism, having favorable effects on tissue repair. Recurrent ulcers can be successfully treated by the intermittent, controlled applications of compressions using a pneumatic pump. Local administration of the vitamin E-based gel also proved useful, and topical application of lidocaine was effective for pain (12). Local administration of vitamin $\mathrm{E}$ has shortened the healing time of digital ulcers from 21 weeks to a mere 13 weeks and also appears to be effective for pain management, at a lower cost of treatment (12). Using the iontophoresis technique, a synthetic prostacyclin analog, treprostinil, can be applied. Low-intensity electric currents produce local vasodilation improving the cutaneous blood flow and healing digital ulcers. Autologous adipose tissue transplantation is a new therapy which registers favorable results (12). However, physiotherapy and psychotherapy have also been demonstrated to be effective $(17,24)$.

In 2000, intravenous immunoglobulins proved to be effective in gastrointestinal and skin involvement by SSc, albeit further studies are necessary to study their benefit (2). Rapidly progressive disease sets can benefit from stem cell autologous transplantation; the cells are taken from the hematogenous bone marrow and the treatment has had extremely favorable results $(2,6)$. ASTIS, a large study conducted in Europe, and the American studies SCOT and ASSIST, revealed that autologous hematopoietic stem cell transplantation increased patient survival rates, as compared to cyclophosphamide, with no adverse events. However, the benefit of this transplantation method was revealed two years after the transplant, being associated with a mortality rate of $10 \%$ (6).

\section{Treatment of skin fibrosis}

Findings of previous studies revealed that methotrexate can have a favorable effect on skin induration, used in doses of $15 \mathrm{mg} /$ week, for more than a year, in spite of the possible adverse reactions, including hepatic toxicity, pulmonary fibrosis, cytopenia and teratogenicity. However, methotrexate is not effective in visceral fibrosis $(4,26,27)$. The antifibrotic action of several immunosuppressants, including MMF, metotrexate and cyclophosphamide, appears to be similar according to previous findings (6). However, more studies are needed to support the antifibrotic action with certainty (4).

Investigations on experimental SSc models have demonstrated the angiotensin-induced profibrotic effect that stimulates TGF- $\beta 1$ synthesis and the excessive extracellular matrix accumulation. However, the antifibrotic effect induced by ACE inhibitors is not sufficiently supported by studies (17). Pruritus, associated with skin fibrosis, and sclerodermal faces, are two aspects of the disease that are difficult to control (6). 


\section{Treatment of pulmonary fibrosis}

Nintedanib, an antifibrotic recommended by current guidelines in the treatment of idiopathic pulmonary fibrosis, is effective against pulmonary fibrosis in SSc. Nintedanib has an antifibrotic effect on the lung by blocking fibroblast growth factor receptor 3 (FGFR3) and platelet-derived growth factor (PDGF) and vascular endothelial growth factor (VEGF) receptors. The combination of nintedanib and azathioprine amplifies the antifibrotic effect and improves forced vital capacity (FVC) by intercepting FGFR3, PDGF and VEGF receptors. Imatinib is also another antifibrotic agent that is currently being approved for patient use (2).

Pirfenidone is a recently approved antifibrotic agent with the same recommendations as nintedanib, being used in secondary and idiopathic pulmonary fibrosis, but for which several studies are needed in order to establish its place among other antifibrotic agents used in SSc (6). A new molecule, lenabasum, which has agonist actions on the cannabinoid receptor type 2 (CB2), is currently under study and appears to be effective in diffuse SSc (2).

Konma and colleagues (11) revealed that tacrolimus administered orally and combined with prednisolone, has mediocre benefits in the interstitial pneumonitis caused by SSc. The study included 11 patients who received a daily dose of $3 \mathrm{mg}$ tacrolimus and prednisolone in doses of $0.5 \mathrm{mg} / \mathrm{kg}$. Of these, only 3 patients exhibited improvement of the respiratory parameters, while 7 patients were stationary, and 1 patient had an unfavorable outcome with worsening respiratory function. Only 2 patients had infectious complications with cytomegalovirus and herpes zoster, complications which were induced by double immunosuppression with tacrolimus and glucococorticoids, while 4 patients progressed to renal impairment after 1 year of treatment with tacrolimus and prednisolone. Survival after one year of therapy was $100 \%$ (11). Biological therapy with rituximab, although currently under research, appears to have beneficial effects in severe cases of pulmonary fibrosis (6).

\section{Treatment of sclerodermal renal crisis}

SRC registers a poor prognosis and is the most severe complication of SSc. Corticosteroids may precipitate SRC. Previous findings have shown progression to SRC in $66 \%$ of patients following a 3 -month corticosteroid therapy and in $33 \%$ of patients treated with $15 \mathrm{mg}$ /day of prednisone $(4,28)$. Patients treated with ACE inhibitors, such as captopril and enalapril, experienced renal function recovery and had increased survival rates after one year of treatment $(29,30)$.

Although ACE inhibitors are often indicated in SRC, the risk of kidney complications cannot be ignored. Authors provide divergent views on the use of ACE inhibitors in $\mathrm{SSc}$-associated hypertension, prior to an SRC episode. Therefore, the risk factors for SRC remain unclear. The observations made by Bütikofer and his collaborators (29) are contradictory with those of other studies regarding the risk of $\mathrm{SRC}$ in SSc-associated hypertension after the administration of ACE inhibitors. Glucocorticoids can also trigger SRC by reducing renal blood flow due to decreased prostaglandin synthesis when the renin-angiotensin-aldosterone system is activated. However, glucocorticoids provide a clear benefit to cutaneous and pulmonary fibrosis (31). Diuretics and $\beta$-blockers are contraindicated as hypotensive medication in SSc as they further limit peripheral blood flow in these patients suffering from vasculopathy. The vasodilating and hypotensive action of calcium blockers is favorable in Raynaud's phenomenon. Calcium channel blockers have the disadvantage of reducing the tone of esophageal muscles and that of the esophageal sphincter. However, there is no evidence of the involvement of calcium blockers in gastroesophageal reflux. The study by Bütikofer et al (29) suggested that the benefit of angiotensin receptor blockers (ARBs) is sufficient for it to be considered as an alternative to ACE inhibitors. Patients with SRC have elevated endothelin levels in the kidneys. Therefore, the vasodilating effect of endothelin receptor antagonists counteracts the existing renal vasoconstriction (31).

Early diagnosis can improve patient prognosis, and ACE inhibitors are the first-line treatment in SRC. The timing of kidney transplantation can be delayed because kidney function can recover over time, even as early as 2 years (32). Immunosuppressants are indicated after grafting in order to prevent recurrent renal attacks (6).

\section{Treatment of esogastrointestinal damage}

The presence of gastroesophageal reflux requires caution, knowing that aspiration of gastric contents into the airways exacerbate respiratory dysfunction (6). The use of proton pump inhibitors is not limited to the management of gastroesophageal reflux but can also be used for treating ulcerative lesions and esophageal stenoses. Intestinal dysmotility complicated with chronic intestinal pseudo-obstruction, abdominal flatulence, dysphagia and early satiety can be controlled by using prokinetics $(4,33)$. Of these, cisaprid has been shown to be effective in gastric emptying but has the disadvantage of inducing cardiac arrhythmias by prolonging the QT segment on the electrocardiogram, thus limiting its use (4). In intestinal bacterial overpopulation it is necessary to rotate broad-spectrum antibiotics such as fluoroquinolones and amoxicillin, with clavulanic acid $(4,33)$. In chronic intestinal pseudo-obstruction, alprostadil, an analogue of prostaglandin E1, has the ability to stimulate smooth muscle fibers inside the intestinal wall (3). The usefulness of probiotics in intestinal overcrowding and their importance in limiting the risk of malnutrition is well known. It is highly recommended to reduce the intake of lactose and fructose as it helps promote fermentation (2).

Pyridostigmine also relieves refractory and recurrent constipation. Less fermenting oligosaccharides can be included in the diet in order to reduce digestive symptoms in combination with probiotics. Surgical treatment may be an alternative when drug treatment remains ineffective (2). A study by Matsumoto et al revealed that long-acting intravenous cyclophosphamide may be useful when endoscopic hemostasis of ectasiated vessels in the gastric antrum fails. The benefit is notable given the possibility of excluding blood transfusion due to post-hemorrhagic anemia (7). N-acetyl cysteine is recommended in autoimmune diseases for its antioxidant effect (6). Lenabasum is a new molecule with agonist action on $\mathrm{CB} 2$ receptors that promises favorable results (2). Looking ahead, research is geared towards new innovative 
treatment opportunities, including vascular repair, starting from autologous endothelial progenitor cells (EPCs) (34). EPCs are cells that have the ability to differentiate into mature vascular endothelial cells and which play a role in angiogenesis and vasculogenesis. Although angiogenesis involves the formation of new blood vessels from existing vascular system, vasculogenesis is the formation of new networks of blood vessels $(34,35)$. The transplantation of EPCs performed by Chong and colleagues (34) was followed by the progression of cellular engineering through blood vessel repair (35).

\section{Conclusions}

Although some aspects of the etiopathogenesis remain unknown, significant progress has been made over the past years that has led to increased survival rates and targeted treatment for the use of new molecules in the pathogenic chain of SSc (1). In recent decades, SSc and other autoimmune diseases and neoplasms have been the focus of numerous studies that have made important contributions to the therapeutic approach (36-38). The aim of future studies is to reveal new facets of the etiopathogenesis of the disease in order to profile future effective therapies for this collagenosis (2), thereby benefitting patients.

\section{Acknowledgements}

The current review was supported by the 'Dunarea de Jos' University of Galati, Romania, through the research center, Multidisciplinary Integrated Center of Dermatological Interface Research MIC-DIR (Centrul Integrat Multidisciplinar de Cercetare de Interfata Dermatologica - CIM-CID).

\section{Funding}

The current review received funding through the 'Dunarea de Jos' University of Galati, Romania, through an internal grant (grant no. RF3668/01.10.2021).

\section{Availability of data and materials}

The analyzed data sets generated during the present study are available from the corresponding author on reasonable request.

\section{Authors' contributions}

$\mathrm{CB}, \mathrm{EN}, \mathrm{MC}, \mathrm{CO}, \mathrm{SF}$ and LGS were involved in the conception of the study and had major contribution in the writing and revising of the manuscript. ALT, DV, LS, AVI, CLM, MLD, $\mathrm{ML}, \mathrm{AN}$ and BIS assisted in the acquisition, analysis and interpretation of the data. All authors have read and approved the final manuscript. Data authentication is not applicable.

\section{Ethics approval and consent to participate}

Not applicable.

\section{Patient consent for publication}

Not applicable.

\section{Competing interests}

The authors declare that they have no competing interests.

\section{References}

1. Sierra-Sepúlveda A,Esquinca-GonzálezA,Benavides-SuárezSA Sordo-Lima DE, Caballero-Islas AE, Cabral-Castañeda AR and Rodríguez-Reyna TS: Systemic sclerosis pathogenesis and emerging therapies, beyond the fibroblast. Biomed Res Int 2019: 4569826, 2019.

2. Barsotti S, Orlandi M, Codullo V, Di Battista M, Lepri G, Della Rossa A and Guiducci S: One year in review 2019: Systemic sclerosis. Clin Exp Rheumatol 37 (Suppl 119): S3-S14, 2019.

3. Juche A, Siegert E, Mueller-Ladner U, Riemekesten G, Günther C, Kötter I, Henes J, Blank N, Voll RE, Ehrchen J, et al: Reality of inpatient vasoactive treatment with prostacyclin derivatives in patients with acral circulation disorders due to systemic sclerosis in Germany. Z Rheumatol 79: 1057-1066, 2020 (In German).

4. Kowal-Bielecka O, Landewé R, Avouac J, Chwiesko S, Miniati I, Czirjak L, Clements P, Denton C, Farge D, Fligelstone K, et al: EULAR recommendations for the treatment of systemic sclerosis: A report from the EULAR scleroderma trials and research group (EUSTAR). Ann Rheum Dis 68: 620-628, 2009.

5. Bobeica C, Niculet E, Halip AI, Gheuca-Solovastru L, Draganescu ML, Popescu IA, Onisor C, Chirobocea S, Lungu M and Craescu M: Predictive value of immunological markers in systemic sclerosis. Exp Ther Med 22: 994, 2021.

6. Denton CP: Advances in pathogenesis and treatment of systemic sclerosis. Clin Med (Lond) 16: 55-60, 2016.

7. Matsumoto Y, Hayashi H, Tahara K, Yasuda T, Tsubouchi S, Yamamoto Y, Mizuuchi T, Mori H and Sawada T: Intravenous cyclophosphamide for gastric antral vascular ectasia associated with systemic sclerosis refractory to endoscopic treatment: A case report and review of the pertinent literature. Intern Med 58: 135-139, 2019.

8. Lynch JP III and McCune WJ: Immunosuppressive and cytotoxic pharmacotherapy for pulmonary disorders. Am J Respir Crit Care Med 155: 395-420, 1997.

9. Konma J, Kotani T, Shoda T, Suzuka T, Fujiki Y, Nagai K, Hata K, Yoshida S, Takeuchi T, Makino S and Arawaka S: Efficacy and safety of combination therapy with prednisolone and oral tacrolimus for progressive interstitial pneumonia with systemic sclerosis: A retrospective study. Mod Rheumatol 28: 1009-1015, 2019.

10. Hughes M and Herrick AL: Digital ulcers in systemic sclerosis. Rheumatology (Oxford) 56: 14-25, 2017.

11. Khor CG, Chen XL, Lin TS, Lu CH and Hsieh SC: Rituximab for refractory digital infarcts and ulcers in systemic sclerosis. Clin Rheumatol 33: 1019-1020, 2014.

12. Beckett VL, Conn DL, Fuster V, Osmundson PJ, Strong CG, Chao EY, Chesebro JH and O'Fallon WM: Trial of plateletinhibiting drug in scleroderma. Double-blind study with dipyridamole and aspirin. Arthritis Rheum 27: 1137-1143, 1984.

13. Bobeică C, Tatu AL, Crăescu M and Solovăstru L: Dynamics of digital ulcers in systemic sclerosis. Exp Ther Med 20: 61-67. 2020.

14. Denton CP, Howell KS, Stratton RJ and Black CM: Long-term low molecular weight heparin therapy for severe Raynaud's phenomenon: A pilot study. Clin Exp Rheumatol 18: 499-502, 2000.

15. Haustein UF: Systemic sclerosis-scleroderma. Dermatol Online J 8: 3, 2002.

16. Goodfield MJ and Rowell NR: Treatment of peripheral gangrene due to systemic sclerosis with intravenous pentoxifylline. Clin Exp Dermatol 14: 161-162, 1989.

17. Berman B and Duncan MR: Pentoxifylline inhibits the proliferation of human fibroblasts derived from keloid, scleroderma and morphea skin and their production of collagen, glycosaminoglycans and fibronectin. Br J Dermatol 123: 339-346, 1990.

18. Fiori G, Marzi T, Bartoli F, Bruni C, Ciceroni C, Palomba M, Zolferino M, Corsi E, Galimberti M, Moggi Pignone A, et al: The challenge of pet therapy in systemic sclerosis: Evidence for an impact on pain, anxiety, neuroticism and social interaction. Clin Exp Rheumatol 36 (Suppl 113): 135-141, 2018.

19. Thakkar V, Nikpour M, Stevens WM and Proudman SM: Prospects for improving outcomes in systemic sclerosis-related pulmonary hypertension. Intern Med J 45: 248-254, 2015. 
20. Channick RN, Simonneau G, Sitbon O, Robbins IM, Frost A, Tapson VF, Badesch DB, Roux S, Rainisio M, Bodin F and Rubin LJ: Effects of the dual endothelin-receptor antagonist bosentan in patients with pulmonary hypertension: A randomized placebo-controlled study. Lancet 358: 1119-1123, 2001.

21. Rubin LJ, Badesch DB, Barst RJ, Galie N, Black CM, Keogh A, Pulido T, Frost A, Roux S, Leconte I, et al: Bosentan therapy for pulmonary arterial hypertension. N Engl J Med 346: 896-903, 2002.

22. Rebegea L, Firescu D, Baciu G and Ciubara A: Psycho-oncology support. BRAIN 10: 77-88, 2019.

23. Solovăstru LG, Stîncanu A, De Ascentii A, Capparé G, Mattana P and Vâță D: Randomized, controlled study of innovative spray formulation containing ozonated oil and $\alpha$-bisabolol in the topical treatment of chronic venous leg ulcers. Adv Skin Wound Care 28: 406-409, 2015.

24. van den Hoogen FH, Boerbooms AM, Swaak AJ, Rasker JJ, van Lier HJ and van de Putte LB: Comparison of methotrexate with placebo in the treatment of systemic sclerosis: A 24-week randomized double-blind trial, followed by a 24 -week observational trial. Br J Rheumatol 35: 364-372, 1996.

25. Arakawa H, Yamasaki M, Kurihara Y, Yamada $H$ and Nakajima Y: Methotrexate induced pulmonary injury: Serial CT findings. J Thorac Imaging 18: 231-236, 2003.

26. Steen VD and Medsger TA Jr: Case-control study of corticosteroids and other drugs that either precipitate or protect from the development of renal scleroderma crisis. Arthritis Rheum 41 1613-1619, 1998.

27. Steen VD, Costantino JP, Shapiro AP and Medsger TA Jr: Outcome of renal crisis in systemic sclerosis: Relation to availability of angiotensin converting enzyme (ACE) inhibitors. Ann Intern Med 113: 352-357, 1990.

28. Steen VD and Medsger TA Jr: Long-term outcomes of renal scleroderma crisis. Ann Intern Med 133: 600-603, 2000.

29. Bütikofer L, Varisco PA, Distler O, Kowal-Bielecka O, Allanore Y, Riemekasten G, Villiger PM and Adler S; EUSTAR collaborators: ACE inhibitors in SSc patients display a risk factor for scleroderma renal crisis-a EUSTAR analysis. Arthritis Res Ther 22: 59, 2020
30. Tyndal AJ, Bannert B, Vonk M, Airò P, Cozzi F, Carreira PE, Bancel DF, Allanore Y, Müller-Ladner U, Distler O, et al: Causes and risk factors of death in systemic sclerosis: A study from the EULAR scleroderma trials and research (EUSTAR) database. Ann Rheum Dis 69: 1809-1815, 2010.

31. Gasbarrini A, Lauritano EC, Gabrielli M, Scarpellini E, Lupascu A, Ojeti V and Gasbarrini G: Small intestinal bacterial overgrowth: Diagnosis and treatment. Dig Dis 25: 237-240, 2007.

32. Chong MS, Ng WK and Chan JK: Concise review: Endothelial progenitor cells in regenerative medicine: Applications and challenges. Stem Cells Transl Med 5: 530-538, 2016.

33. Sukmawati D and Tanaka R: Introduction to next generation of endothelial progenitor cell therapy: A promise in vascular medicine. Am J Transl Res 7: 411-421, 2015.

34. Tatu AL and Ionescu MA: Multiple autoimmune syndrome type III-thyroiditis, vitiligo and alopecia areata. Acta Endocrinol (Buchar) 13: 124-125, 2017.

35. Nwabudike LC, Elisei AM, Buzia OD, Miulescu M and Tatu AL: Statins. A review on structural perspectives, adverse reactions and relations with son-melanoma skin cancer. Rev Chim (Buchar) 69: 2557-2562, 2018.

36. Mihăilă B, Dinică RM, Tatu AL and Buzia OD: New insights in vitiligo treatments using bioactive compounds from Piper nigrum. Exp Ther Med 17: 1039-1044, 2019.

37. Zheng JN, Yang QR, Zhu GQ, Pan L, Xia JX and Wang Q: Comparative efficacy and safety of immunosuppressive therapies for systemic sclerosis related interstitial lung disease: A Bayesian network analysis. Mod Rheumatol 30: 687-695, 2020.

38. Gonzalez-Nieto JA,Martin-Suares IJ and Gil-Munoz FL: Eficacia del micofenolato asociado a metotrexato como tratamiento de mantenimiento de la enfermedad pulmonary intersticial asociada a la sclerosis sistémica. Arch Bronconeumol 47: 575, 2011. International (CC BY-NC-ND 4.0) License. 